\title{
VIRAL HEPATITIS
}

\section{New advances in IL28B genetics}

Although new antiviral agents are available to treat chronic HCV infection-a major cause of cirrhosis and hepatocellular carcinoma (HCC) - the success rate is less than optimal and intensive research efforts have focused on understanding the association of IL28B polymorphisms with spontaneous clearance of HCV infection and response to therapy. Two studies, published in Hepatology and The Journal of Experimental Medicine, respectively, now provide new insights into this association and could have important implications for the clinical management of patients with chronic hepatitis $\mathrm{C}$.

The first study, conducted by Marc Ghany and colleagues, aimed to deterime whether the IL28B genotype affects fibrosis progression and clinical outcomes (including hepatic encephalopathy, bleeding varices and $\mathrm{HCC}$ ) in patients with chronic hepatitis $\mathrm{C}$.

An analysis of baseline biopsy samples from 1,483 patients revealed that those with IL28B CC genotype (rs12979860) had significantly greater levels of portal inflammation and alanine aminotransferase (ALT) than those with non-CC genotypes $(P<0.05)$. A paired biopsy analysis (including 276 of these patients) revealed no statistically significant difference in the frequency of fibrosis progression between patients with CC or non-CC genotypes (17\% versus 23\%). Indeed, from logistic regression analysis, only increased alkaline phosphatase levels at baseline, reduced numbers of platelets or increased levels of hepatic steatosis were associated with fibrotic progression. Of note, patients with the CC genotype were more likely to develop adverse clinical outcomes than non-CC individuals (32\% versus $16 \%$ ).

"Our most significant findings are that the IL28B CC genotype is associated with increased levels of hepatic necroinflammation and ALT, but not fibrosis progression," states Ghany. "Perhaps more importantly, IL28B CC genotype was associated with worse clinical outcomes in patients with chronic hepatitis $C$," he adds. The researchers propose that the CC genotype results in a state of enhanced immunity that can act in two ways: to promote viral clearance on the one hand, but also increase necroinflammation and hepatic decompensation on the other.

Further studies are required to "examine the effect of suppressing inflammation on the rate of clinical outcomes and also to explore the profibrotic pathways that are independent of inflammation," Ghany concludes.

The second study, by Pierre-Yves Bochud and co-workers, reports the identification of a new polymorphism in the CpG region upstream of IL28B that is a better predictor of HCV clearance than rs12979860.

Analysis of the methylation pattern in this region revealed the presence of a $T$ deletion followed by a $T>G$ substitution that introduces a new methylation site. The researchers went on to analyse peripheral blood mononuclear cells isolated from patients with different allelic combinations of both polymorphisms. "By genotyping $T T /-G$ in treatment responders and non-responders, we were able to determine that TT/-G was a better marker of HCV clearance than rs12979860," Bochud explains. The researchers demonstrated that induction of IL28B and IP-10 (also know as CXCL10) mRNA was reliant on the presence of the TT/-G polymorphism, but not that of $r$ s 12979860 . Therefore, TT/-G is the only variant that has had confirmed functional effects so far.

The researchers postulate that this further level of understanding of IL28B genetic regulation could have implications for patient management as clinicians could start to conduct TT/-G genotyping. Indeed "genetic testing might help to design personalized treatment strategies for patients with chronic hepatitis C," he says.

Katherine Smith

Original articles Noureddin, M. et al. Association of IL28B genotype with fibrosis progression and clinical outcomes in patients with chronic hepatitis C: A longitudinal analysis. Hepatology doi:10.1002/ hep.26506 | Bibert, S. et al. IL28B expression depends on a novel TT/-G polymorphism which improves HCV clearance prediction. J. Exp. Med. doi:10.1084/jem.20130012. 